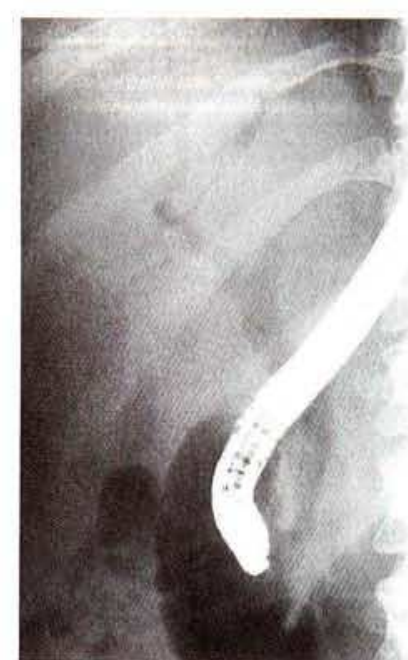

Figure 1: An air cholangiogram in a patient who had previously undergone endoscopic sphincterotomy

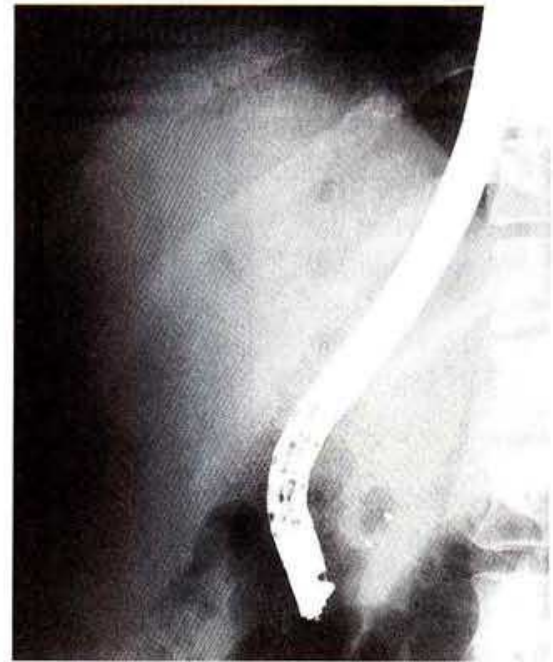

Figure 2: Aspirating the air with a doublelumen balloon occluding the distal common bile duct.

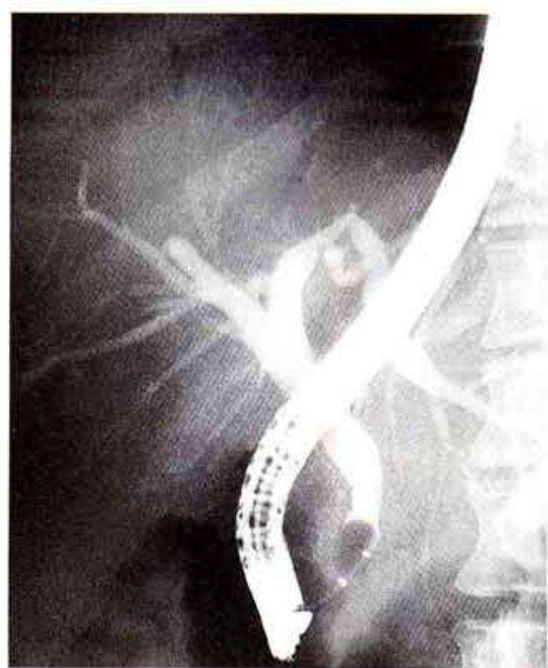

Figure 3: A good-quality balloon occlusion ERC is obtaine after the complete removal of the air.

\section{Improving the Quality of Balloon Occlusion Cholangiography by Identifying and Deflating Air Cholangiograms}

Choledochoduodenostomy or sphincterotomy may reduce the quality of endoscopic retrograde cholangiography (ERC) by preventing adequate filling of the biliary system with contrast, and by allowing air-contrast mixing. Occlusion of the distal common bile duct by balloon before injection of contrast is a recognized method of improving cholangiography in such cases, but does not solve the problem of air already present in the biliary system. Our policy for these cases is to screen before the injection of contrast, looking specifically for an air cholangiogram. If present, the air can be aspirated completely through a standard double-lumen balloon placed and inflated at the lower end of the common bile duct. When complete deflation is confirmed radiologically, it is possible to obtain high-quality ERC with the ballon still in place, without false-positive filling defects due to air. Although this technique is simple and effective, we have not seen it described elsewhere.

\section{W. Dickey, K. G. Porter}

Department of Gastroenterology, Belfast City Hospital and Department of Medicine, Queen's University of Belfast, Belfast, Northern Ireland

Corresponding Author

W. Dickey, M.D.

Department of Medicine Institute of Clinical Science

Queen's University of Belfast

Grosvenor Road

Belfast BT12 6BJ

United Kingdom 